\title{
Clinical implication of metabolic syndrome in nonobese patients with antineutrophil cytoplasmic antibody- associated vasculitis
}

\section{Soo Bin soobinl22@naver.com}

Yonsei University College of Medicine

Hyeok Chan Kwon

Dankook University College of Medicine

Jung Yoon Pyo

Yonsei University College of Medicine

Mi II Kang

Dankook University College of Medicine

Jason Jungsik Song

Yonsei University College of Medicine

Yong-Beom Park

Yonsei University College of Medicine

Jun Yong Park

Yonsei University College of Medicine

Sang-Won Lee ( $\nabla$ sangwonlee@yuhs.ac)

Yonsei University College of Medicine https://orcid.org/0000-0002-8038-3341

\section{Research article}

Keywords: Metabolic syndrome, antineutrophil cytoplasmic antibody-associated vasculitis, cardiovascular disease, prevalence, risk

Posted Date: May 19th, 2020

DOI: https://doi.org/10.21203/rs.3.rs-28821/v1

License: (c) (1) This work is licensed under a Creative Commons Attribution 4.0 International License. Read Full License 


\section{Abstract}

Objective: We investigated the prevalence of metabolic syndrome (MetS) in all or nonobese patients with antineutrophil cytoplasmic antibody (ANCA)-associated vasculitis (AAV) and compared it with age- and gendermatched controls. Also, we assessed the effect of variables at diagnosis on the risk of cardiovascular disease (CVD) in all or nonobese AAV patients.

Methods: In this study, 173 AAV patients and 344 controls were included and MetS was defined by the National Cholesterol Education Program Adults Treatment Panel III criteria. The obesity based on BMI was defined as BMI $\geq 25 \mathrm{~kg} / \mathrm{m} 2$. The follow-up duration was defined as the period from diagnosis to the last visit or to each poor outcome occurrence.

Results: The median age of AAV patients was 58.7 years and 57 patients were men. The prevalence of MetS was $50.9 \%$ in all AAV patients and $46.5 \%$ in nonobese AAV patients, which were significantly higher than $37.8 \%$ in all controls and $28.2 \%$ in nonobese controls. In the Kaplan-Meier survival analysis, Mets at diagnosis significantly reduced the cumulative CVD-free survival rate in both all and nonobese AAV patients. In the multivariable Cox hazards model analysis, CVD during follow-up was significantly associated with both BVAS (HR 1.159) and MetS at diagnosis (HR 9.036) in nonobese AAV patients.

Conclusions: The prevalence of MetS at diagnosis in all or nonobese AAV patients was significantly higher than those in all or nonobese controls. Furthermore, both BVAS and MetS at diagnosis increased the risk of CVD in nonobese AAV patients.

\section{Introduction}

The concept of metabolic syndrome (MetS) is defined by the constellation of metabolic abnormalities that confer to the increased risk of cardiovascular disease (CVD), type 2 diabetes mellitus and all-cause morbidity and mortality [1, 2]. So far, several definitions of MetS, such as the World Health Organization (WHO) definition and the European Group for the Study of Insulin Resistance definition, have been proposed [3, 4]. The National Cholesterol Education Program Adults Treatment Panel III criteria for MetS (the 2005 NCEP-ATP-III criteria) is currently used for the classification of MetS: insulin resistance, obesity (waist circumference), hyperlipidaemia, glucose intolerance, and hypertension have been recognized and accepted as the fundamental mechanisms [5, 6]. Among these mechanisms, insulin resistance is the most important contributor and it is associated with vascular thrombosis- and inflammation-related factors, such as lipoprotein dysregulation, prothrombotic changes, low-grade inflammatory conditions and vascular dysfunction [1]. In particular, MetS has been proved to be associated with proinflammatory cytokines including tumour necrosis factor (TNF)-a, interleukin (IL)-1 and IL6, which can often participate in and accelerate the process of atherosclerosis and thrombosis [7]. Therefore, the primary concern regarding the systemic complication of MetS is the risk of CVD [2].

Recently, the interlink between the metabolic and immune systems has been a global emerging interest. The role of the immune system in maintaining metabolic homeostasis has been implicated through many studies and is now acknowledged that the disturbance in the immune-metabolic interaction may result in abnormal metabolic states, culminating in metabolic diseases such as MetS [8]. So far, there have been several studies investigating the association of MetS with autoimmune rheumatic diseases and the increased prevalence of MetS in patients with autoimmune rheumatic diseases such as systemic lupus erythematosus, rheumatoid arthritis and vasculitis 
have been reported. Especially in patients with systemic vasculitis, the prevalence of MetS was high, and consequentially to CVD as well [9].

Anti-neutrophil cytoplasmic antibody (ANCA)-associated vasculitis (AAV) is a group of systemic necrotizing vasculitis which primarily affects the small-sized vessels and occasionally the medium-sized ones and consists of three subtypes such as microscopic polyangiitis (MPA), granulomatosis with polyangiitis (GPA) and eosinophilic granulomatosis with polyangiitis (EGPA) $[10,11]$. Since chronic low-grade inflammation of AAV may provoke insulin resistance and disturb metabolic homeostasis, leading to MetS, it can be theoretically assumed that the prevalence of Mets can be gradually increased in AAV patients. Based on this assumption, a previous study conducted in the UK, which compared the prevalence of Mets between AAV patients and controls, reported a higher proportion of MetS in AAV patients compared to controls [12]. MetS may have an influence on the poor outcomes of AAV: the high risk of CVD has been reported in AAV, which, in turn, has led to the revision of the European League Against Rheumatism (EULAR) guideline for periodic CVD risk evaluation in AAV patients [1315]. In addition, the effect of MetS on the risk of CVD might be clearer in nonobese people [16]. However, to our knowledge, there was no study sufficiently investigating whether Mets at diagnosis could increase the risk of CVD during follow-up in AAV patients. Hence, in this study, we investigated and compared the prevalence of Mets at diagnosis between AAV patients and age- and gender-matched controls, and furthermore between nonobese AAV patients and controls who had body mass index $(B M I)<25 \mathrm{~kg} / \mathrm{m}^{2}$ [17]. Also, we investigated whether MetS at diagnosis could increase the risk of CVD and other poor outcomes of AAV during follow-up in both all AAV patients and nonobese AAV patients.

\section{Patients And Methods}

\section{Patients}

We included 173 patients with AAV, who were reclassified as AAV based on the 2007 European Medicines Agency algorithm for AAV and polyarteritis nodosa and the 2012 revised International Chapel Hill Consensus Conference Nomenclature of Vasculitides and we reviewed their medical records. All patients were initially diagnosed as AAV at the Division of Rheumatology, the Department of Internal Medicine, Yonsei University College of Medicine, Severance Hospital, from October 2000 to March 2019. They all had well-documented medical records with which clinical and laboratory data that reviewed ANCA positivity and both Birmingham vasculitis activity score (BVAS) version 3 and five-factor score (FFS) were calculated at diagnosis $[18,19]$. AAV patients, who had serious medical conditions mimicking AAV or enabling ANCA false-positivity, such as chronic liver diseases, coexisting malignancies, serious infections, and drugs at the time of diagnosis, were excluded from this study. For controls, the medical records of general people, who had consecutively visited Severance Executive Healthcare Clinic in Severance Hospital, a university-affiliated tertiary care hospital, for a comprehensive medical health check-up, were also reviewed [20]. And, 344 age- and gender-matched people without any serious medical condition were included in this study as controls. The cross-sectional diseases at diagnosis or the poor outcomes of AAV during follow-up were identified by the 10th revised International Classification Diseases (ICD-10) and drugs being administered were confirmed using the Korean Drug Utilization Review (DUR) system. This study was approved by the Institutional Review Board (IRB) of Severance Hospital (4-2017-0673 for AAV patients and 4-2018-0856 for controls), who waived the need for the written informed consent, as this was a retrospective study.

\section{The 2005 NCEP-ATP-III criteria}


The 2005 NCEP-ATP-III criteria consist of five components: i) central obesity based on waist circumference for Asian countries (men $\geq 90 \mathrm{Cm}$ and women $\geq 80 \mathrm{Cm}$ ); ii) hypertension (blood pressure $\geq 130 / 85 \mathrm{mmHg}$ ); iii) hypertriglyceridemia (triglyceride $\geq 150 \mathrm{mg} / \mathrm{dL}$ ); iv) low high-density lipoprotein (HDL)-cholesterol (men < $40 \mathrm{mg} / \mathrm{dL}$ and women $<50 \mathrm{mg} / \mathrm{dL}$ ) and v) Impaired glucose tolerance (fasting glucose $\geq 100 \mathrm{mg} / \mathrm{dL}$ ) or type 2 diabetes mellitus $[6,12,21]$.

\section{Variables at diagnosis and during follow-up}

In terms of the variables at diagnosis, age, male gender and BMI were obtained. In the general Korean population, obesity based on BMI was defined as $B M I \geq 25 \mathrm{~kg} / \mathrm{m}^{2}$ [17], and thus, in this study, nonobese patients and controls were defined as those having BMI $<25 \mathrm{~kg} / \mathrm{m}^{2}$. AAV subtypes, ANCA positivity and both BVAS and FFS were reviewed. We reviewed the results for ANCA by both an indirect immunofluorescence assay (perinuclear $(P)$ ANCA and cytoplasmic (C)-ANCA) and antigen-specific assays for ANCA (myeloperoxidase (MPO)-ANCA and proteinase 3 (PR3)-ANCA). In patients who tested positive in the indirect fluorescence assay, but negative in antigen-specific assays, P-ANCA positivity was considered as MPO-ANCA positivity and C-ANCA positivity as PR3-ANCA positivity $[22,23]$. Comorbidities at diagnosis, such as chronic kidney disease, diabetes mellitus, hypertension, dyslipidaemia and interstitial lung disease, were collected. The results of routine laboratory tests at diagnosis were also evaluated. In terms of the variables during follow-up, the poor outcomes of AAV were defined as all-cause mortality, relapse, end-stage renal disease (ESRD), cerebrovascular accident (CVA) and CVD. The follow-up duration was defined as the period between the date of the diagnosis of AAV and the date of the last visit for survived patients. For deceased patients, the follow-up duration based on all-cause mortality was defined as the period between the initial diagnosis of AAV and the time of death. For patients who had any poor outcomes, the follow-up duration based on each poor outcome was defined as the period starting from the diagnosis of AAV until each poor outcome appeared. Also, administered medications were assessed.

\section{Statistical analyses}

All statistical analyses were conducted using the SPSS software (version 23 for Windows; IBM Corp., Armonk, NY, USA). Continuous variables were expressed as a median (interquartile range), and categorical variables were expressed as number and the percentage. Significant differences in categorical variables between the two groups were analysed using the Chi-square and Fisher's exact tests. Significant differences in continuous variables between the two groups were compared using the Mann-Whitney test. The odds ratio (OR) was assessed using the multivariable logistic regression analysis of variables with p-values less than 0.05 in the comparative analysis. The optimal cut-off for BMI in predicting MetS at diagnosis was extrapolated by calculating the receiver operator characteristic (ROC) curve and selecting the maximised sum of the sensitivity and specificity. The relative risk (RR) was analysed using contingency tables and the chi-square test. Each cumulative poor outcomefree survival rate was analysed using the Kaplan-Meier survival analysis. The multivariable Cox hazard model using variables with $\mathrm{p}$-values less than 0.05 in the univariable Cox hazard model was conducted to appropriately obtain the hazard ratios (HRs) during the considerable follow-up duration. P-values less than 0.05 were considered as statistically significant.

\section{Results}

\section{Characteristics of AAV patients}


In regard to variable at diagnosis, the median age of AAV patients was 58.7 years and 57 patients were men. Ninety-seven patients were classified as MPA, 42 patients were classified as GPA and 34 patients were classified as EGPA. MPO-ANCA (or P-ANCA) was detected in 115 patients and ANCA was negative in 35 patients. The most common comorbidity was hypertension $(46.2 \%)$, followed by chronic kidney disease (stage $3-5)(29.5 \%)$ and dyslipidaemia (28.3\%). In regard to variables during the follow-up period, 14 patients died of any cause. The most frequently occurred poor outcome of AAV was relapse (32.4\%), followed by ESRD (19.1\%). Twelve of 173 patients $(6.9 \%)$ had experienced CVD after the diagnosis of AAV. One hundred sixty-two patients had received glucocorticoid (93.6\%) during follow-up. The most common immunosuppressive drug administered was cyclophosphamide (49.7\%), followed by azathioprine (48.0\%) (Table 1 ). 
Table 1

Characteristics of AAV patients with at diagnosis and during follow-up ( $N=173)$

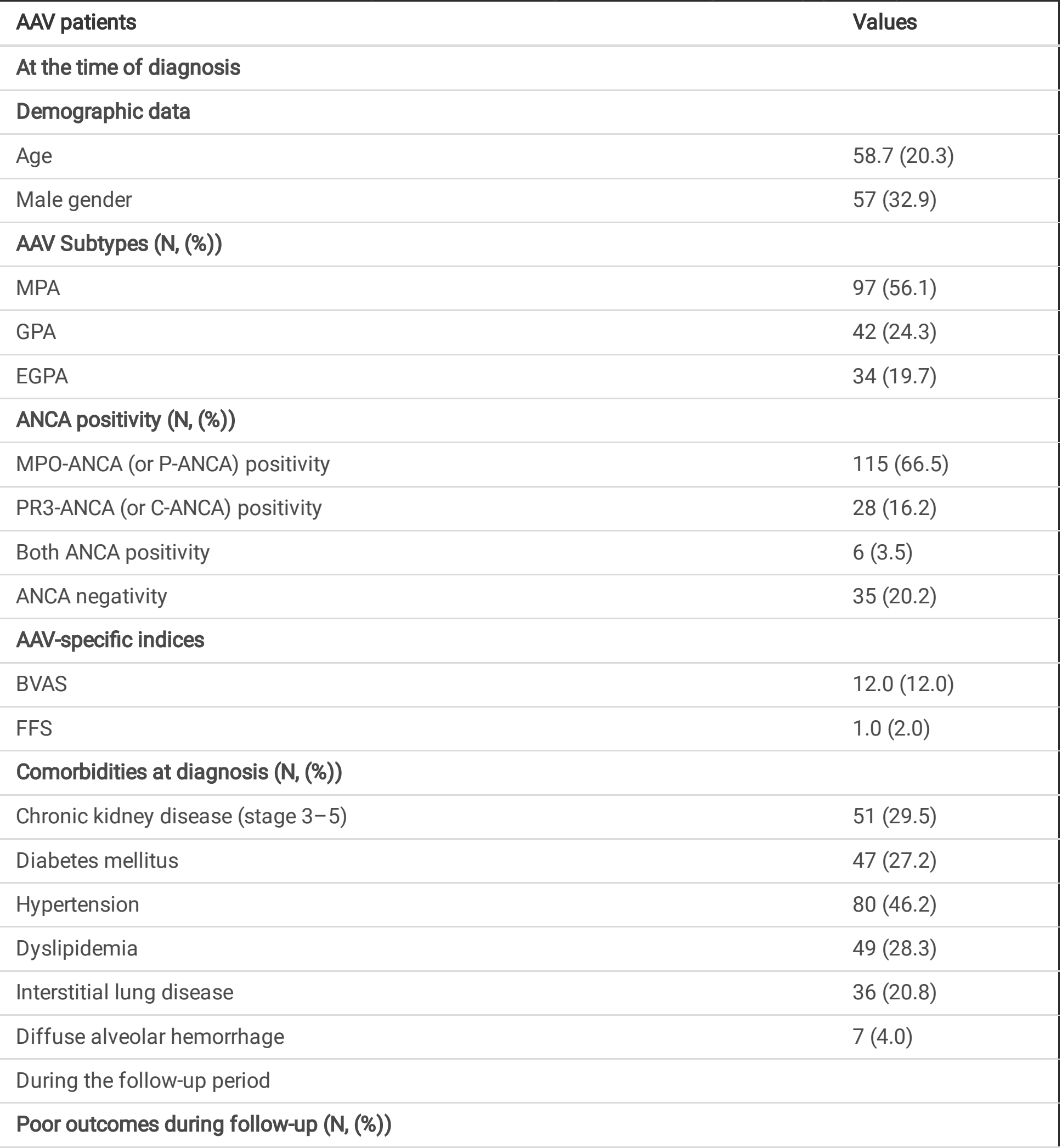

Values are expressed as a median (interquartile range, IQR) or $\mathrm{N}(\%)$.

AAV: antineutrophil cytoplasmic antibody (ANCA)-associated vasculitis; MPA: microscopic polyangiitis; GPA: granulomatosis with polyangiitis; EGPA: eosinophilic granulomatosis with polyangiitis; MPO: myeloperoxidase; P: perinuclear; PR3: proteinase 3; C: cytoplasmic; BVAS: Birmingham vasculitis activity score; FFS: five-factor score; ESRD: end-stage renal disease; CVA: cerebrovascular accident; CVD: cardiovascular disease. 
All-cause mortality

Relapse

ESRD

CVA
$14(8.1)$

$56(32.4)$

$33(19.1)$

$12(6.9)$

$12(6.9)$

CVD

Glucocorticoid

$86(49.7)$

Cyclophosphamide

26 (15.0)

Rituximab

$83(48.0)$

Azathioprine

$22(12.7)$

Mycophenolate mofetil

$10(5.8)$

Tacrolimus

$17(9.8)$

Methotrexate

Values are expressed as a median (interquartile range, IQR) or $\mathrm{N}(\%)$.

AAV: antineutrophil cytoplasmic antibody (ANCA)-associated vasculitis; MPA: microscopic polyangiitis; GPA: granulomatosis with polyangiitis; EGPA: eosinophilic granulomatosis with polyangiitis; MPO: myeloperoxidase; P: perinuclear; PR3: proteinase 3; C: cytoplasmic; BVAS: Birmingham vasculitis activity score; FFS: five-factor score; ESRD: end-stage renal disease; CVA: cerebrovascular accident; CVD: cardiovascular disease.

\section{Comparison of MetS-related variables between 173 AAV patients and 344 controls}

There were no significant differences in the demographic data, in particular, the number of ex-smokers between AAV patients and controls; however, AAV patients exhibited the lower median BMI than controls (22.2 vs.

$23.4 \mathrm{~kg} / \mathrm{m}^{2}$ ). Inversely, the number of AAV patients, who fulfilled the 2005 NCEP-ATP-III criteria, was significantly higher than that of controls who satisfied the same criteria (50.9\% vs. 37.8\%). Among the five components of the 2005 NCEP-ATP-III criteria, AAV patients exhibited central obesity (53.2\% vs. $71.2 \%$ ) less frequently than controls. Meanwhile, they showed hypertriglyceridemia (49.7\% vs. $23.5 \%)$ and impaired glucose tolerance $(60.1 \%$ vs. $24.4 \%)$ more often than controls. In regard to MetS-related laboratory results, AAV patients exhibited the higher median harmful cholesterols than controls (Table 2). 
Table 2

Comparison of MetS-related variables between AAV patients and controls

\begin{tabular}{|c|c|c|c|c|}
\hline Variables & $\begin{array}{l}\text { Total }(\mathrm{N}= \\
517)\end{array}$ & $\begin{array}{l}\text { AAV } \\
\text { patients } \\
(\mathrm{N}=173)\end{array}$ & $\begin{array}{l}\text { Controls } \\
(\mathrm{N}=344)\end{array}$ & $\begin{array}{l}\text { P- } \\
\text { value }\end{array}$ \\
\hline \multicolumn{5}{|l|}{ Demographic data } \\
\hline Age (year old) & $58.0(21.1)$ & $58.7(20.3)$ & $\begin{array}{l}58.0 \\
(21.8)\end{array}$ & 0.720 \\
\hline Male gender (N, (\%)) & $166(32.1)$ & $57(32.9)$ & $\begin{array}{l}109 \\
(31.7)\end{array}$ & 0.772 \\
\hline BMI $\left(\mathrm{kg} / \mathrm{m}^{2}\right)$ & $23.2(3.5)$ & $22.2(4.4)$ & $23.4(3.5)$ & $<.001$ \\
\hline Ex-smoker (N, (\%)) & $40(7.7)$ & $14(8.1)$ & $26(7.6)$ & 0.830 \\
\hline $\begin{array}{l}\text { Fulfillment of NCEP-ATP III } 2005 \text { criteria for MetS (N, } \\
(\%))\end{array}$ & $218(42.2)$ & $88(50.9)$ & $\begin{array}{l}130 \\
(37.8)\end{array}$ & 0.004 \\
\hline \multicolumn{5}{|l|}{2005 NCEP-ATP-III criteria for MetS (N, (\%)) } \\
\hline Waist circumference (male) & $91.6(9.0)$ & $90.3(10.6)$ & $92.0(8.0)$ & 0.039 \\
\hline Waist circumference (female) & $82.3(9.5)$ & $81.0(10.9)$ & $83.1(8.4)$ & $\begin{array}{l}< \\
0.001\end{array}$ \\
\hline Central obesity based on waist circumference & $337(65.2)$ & $92(53.2)$ & $\begin{array}{l}245 \\
(71.2)\end{array}$ & $\begin{array}{l}< \\
0.001\end{array}$ \\
\hline Hypertension & $215(41.6)$ & $80(46.2)$ & $\begin{array}{l}135 \\
(39.2)\end{array}$ & 0.208 \\
\hline Hypertriglyceridemia & $167(32.3)$ & $86(49.7)$ & $81(23.5)$ & $\begin{array}{l}< \\
0.001\end{array}$ \\
\hline Low HDL cholesterol & $211(40.8)$ & $62(35.8)$ & $\begin{array}{l}149 \\
(43.3)\end{array}$ & 0.103 \\
\hline Impaired fasting glucose or type 2 diabetes mellitus & $188(36.4)$ & $104(60.1)$ & $84(24.4)$ & $<.001$ \\
\hline \multicolumn{5}{|l|}{ Laboratory data } \\
\hline Total cholesterol (mg/dL) & $183(57)$ & $174(63)$ & $187(52)$ & 0.017 \\
\hline Triglyceride (mg/dL) & $103(75)$ & $117(73)$ & $100(73)$ & 0.002 \\
\hline HDL cholesterol (mg/dL) & $49(22)$ & $48(25)$ & $49(20)$ & 0.257 \\
\hline
\end{tabular}

Values are expressed as a median (interquartile range, IQR) or N (\%).

MetS: metabolic syndrome; AAV: antineutrophil cytoplasmic antibody (ANCA)-associated vasculitis; BMI: body mass index; NCEP-ATP-III: national cholesterol education program-adult treatment panel III; HDL: highdensity lipoprotein; LDL: low-density lipoprotein; BUN: blood urea nitrogen; eGFR: estimated glomerular filtration rate. 


\begin{tabular}{|c|c|c|c|c|}
\hline Variables & $\begin{array}{l}\text { Total }(\mathrm{N}= \\
517)\end{array}$ & $\begin{array}{l}\text { AAV } \\
\text { patients } \\
(\mathrm{N}=173)\end{array}$ & $\begin{array}{l}\text { Controls } \\
(\mathrm{N}=344)\end{array}$ & $\begin{array}{l}\mathrm{P}- \\
\text { value }\end{array}$ \\
\hline LDL cholesterol (mg/dL) & $100(50)$ & $92(47)$ & $104(48)$ & $\dot{0}_{0.001}$ \\
\hline Fasting glucose (mg/dL) & $96(17)$ & $102(36)$ & $94(14)$ & $\begin{array}{l}< \\
0.001\end{array}$ \\
\hline Creatinine $(\mathrm{mg} / \mathrm{dL})$ & $0.8(0.3)$ & $0.9(1.2)$ & $0.7(0.3)$ & $\begin{array}{l}< \\
0.001\end{array}$ \\
\hline \multicolumn{5}{|c|}{ Values are expressed as a median (interquartile range, IQR) or N (\%). } \\
\hline \multicolumn{5}{|c|}{$\begin{array}{l}\text { MetS: metabolic syndrome; AAV: antineutrophil cytoplasmic antibody (ANCA)-associated vasculitis; BMI: } \\
\text { body mass index; NCEP-ATP-III: national cholesterol education program-adult treatment panel III; HDL: high- } \\
\text { density lipoprotein; LDL: low-density lipoprotein; BUN: blood urea nitrogen; eGFR: estimated glomerular } \\
\text { filtration rate. }\end{array}$} \\
\hline
\end{tabular}

\section{Comparison of MetS-related variables between 144 nonobese AAV patients and 255 nonobese controls}

To minimise the effect of obesity based on BMI on the prevalence of MetS, we compared the prevalence of MetS in nonobese AAV patients and controls who had BMI $<25 \mathrm{~kg} / \mathrm{m}^{2}$. Sixty-seven of 144 AAV patients (46.5\%) exhibited the cross-sectional MetS, whereas only 72 of 255 controls $(28.2 \%)$ had it (RR $2.212, \mathrm{P}<0.001)$. Whereas, among obese AAV patients and controls who had BMI $\geq 25 \mathrm{~kg} / \mathrm{m}^{2}$, the two groups showed the similar prevalence of MetS (Fig. 1).

\section{Comparison of variables at diagnosis between $88 \mathrm{AAV}$ patients with Mets and 85 AAV patients without Mets}

AAV patients were divided into the two groups based on MetS at diagnosis: 88 patients were assigned to the groups of AAV patients with MetS and they showed higher frequency in all five components of the 2005 NCEPATP-III criteria than those without MetS. At the time of diagnosis, AAV patients with MetS were older and more obese than those without MetS: however, no difference in gender and ex-smoker between the two groups was found. In regard to ANCA positivity and AAV-specific inflammatory indices, AAV patients with Mets showed a significantly higher frequency of MPO-ANCA (or P-ANCA) positivity and the higher cross-sectional BVAS and FFS than those without MetS. Among comorbidities, the proportions of chronic kidney disease, diabetes mellitus, hypertension and dyslipidaemia were significantly increased in AAV patients with MetS compared to those without MetS. Among laboratory results, AAV patients with MetS exhibited higher levels in all the variables than those without MetS except for haemoglobin and serum albumin which showed an opposite tendency (Table 3). 
Table 3

Comparison of variables at diagnosis and during follow-up between AAV patients with MetS and those without

AAV patients

AAV patients

AAV patients

with MetS $(\mathbf{N}=$

88)

without MetS $(\mathrm{N}=$

Pvalue

85)

At the time of diagnosis

2005 NCEP-ATP-III criteria for MetS

Waist circumference (male)

$91.8(10.6)$

87.5 (10.7)

0.026

Waist circumference (female)

$84.0(11.7)$

76.9 (8.5)

$<0.001$

Waist circumference (male $\geq 90 \mathrm{~cm}$, female $\geq$

$80 \mathrm{~cm})$

61 (69.3)

31 (36.5)

$<0.001$

Hypertension (> 130/85 mmHg or medication)

60 (68.2)

Triglyceride (>150 mg/dL)

67 (76.1)

$20(23.5)$

$<0.001$

HDL cholesterol (male $<40 \mathrm{mg} / \mathrm{dL}$,

$44(50.0)$

19 (22.4)

$<0.001$

female $<50 \mathrm{mg} / \mathrm{dL}$ )

Impaired fasting glucose $(>100 \mathrm{mg} / \mathrm{dL}$ or

medication)

70 (79.5)

$18(21.1)$

$<0.001$

\section{Demographic data}

Age (year old)

$61.4(14.0)$

53.3 (26.3)

0.002

Male gender $(\mathrm{N},(\%))$

$30(34.1)$

27 (31.8)

0.745

$\operatorname{BMI}\left(\mathrm{kg} / \mathrm{m}^{2}\right)$

23.3 (3.3)

$21.1(3.6)$

$<0.001$

Ex-smoker

9 (10.2)

5 (8.6)

0.405

\section{AAV subtypes ( $N,(\%))$}

MPA

GPA

EGPA

ANCA positivity $(\mathrm{N},(\%))$

MPO-ANCA (or P-ANCA) positivity
47 (53.4)

$23(26.1)$

$18(20.5)$

$66(75.0)$

49 (57.6)

0.016

Values are expressed as a median (interquartile range, IQR) or N (\%).

AAV: antineutrophil cytoplasmic antibody (ANCA)-associated vasculitis; MetS: metabolic syndrome; NCEPATP-III: national cholesterol education program-adult treatment panel III; HDL: high-density lipoprotein; BMI: body mass index; MPA: microscopic polyangiitis; GPA: granulomatosis with polyangiitis; EGPA: eosinophilic granulomatosis with polyangiitis; MPO: myeloperoxidase; P: perinuclear; PR3: proteinase 3; C: cytoplasmic; BVAS: Birmingham vasculitis activity score; FFS: five-factor score; ESR: erythrocyte sedimentation rate; CRP: C-reactive protein; ESRD: end-stage renal disease; CVA: cerebrovascular accident; CVD: cardiovascular disease. 


\begin{tabular}{|c|c|c|c|}
\hline AAV patients & $\begin{array}{l}\text { AAV patients } \\
\text { with MetS ( } \mathrm{N}= \\
\text { 88) }\end{array}$ & $\begin{array}{l}\text { AAV patients } \\
\text { without MetS ( } N= \\
\text { 85) }\end{array}$ & $\begin{array}{l}\mathrm{P}- \\
\text { value }\end{array}$ \\
\hline PR3-ANCA (or C-ANCA) positivity & $12(13.6)$ & $16(18.8)$ & 0.354 \\
\hline Both ANCA positivity & $1(1.1)$ & $5(5.9)$ & 0.113 \\
\hline ANCA negativity & $11(12.5)$ & $24(28.2)$ & 0.252 \\
\hline \multicolumn{4}{|l|}{ AAV-specific indices } \\
\hline BVAS & $14.0(12.0)$ & $11.0(10.0)$ & 0.018 \\
\hline FFS & $1.0(1.0)$ & $1.0(2.0)$ & 0.010 \\
\hline \multicolumn{4}{|l|}{ Comorbidities at diagnosis (N, (\%)) } \\
\hline Chronic kidney disease (stage $3-5$ ) & $34(38.6)$ & $17(20.0)$ & 0.007 \\
\hline Diabetes mellitus & $39(44.3)$ & $8(9.4)$ & $<0.001$ \\
\hline Hypertension & $60(68.2)$ & $20(23.5)$ & $<0.001$ \\
\hline Dyslipidemia & $42(47.7)$ & $7(8.2)$ & $<0.001$ \\
\hline Interstitial lung disease & $20(22.7)$ & $16(18.8)$ & 0.527 \\
\hline \multicolumn{4}{|l|}{ Laboratory results } \\
\hline White blood cell count $\left(/ \mathrm{mm}^{3}\right)$ & $9,230.0(6,570.0)$ & $7,280.0(5,345.0)$ & 0.080 \\
\hline Hemoglobin (g/dL) & $10.7(3.9)$ & $12.1(2.9)$ & 0.004 \\
\hline Platelet count $\left(x 1,000 / \mathrm{mm}^{3}\right)$ & $317.5(197.0)$ & 247. (133.0) & 0.015 \\
\hline Fasting glucose (mg/dL) & $110(46)$ & $95(25)$ & $<0.001$ \\
\hline Creatinine $(\mathrm{mg} / \mathrm{dL})$ & $1.1(2.2)$ & $0.8(0.5)$ & 0.006 \\
\hline Serum albumin $(\mathrm{g} / \mathrm{dL})$ & $3.5(1.1)$ & $3.8(0.9)$ & 0.005 \\
\hline $\mathrm{ESR}(\mathrm{mm} / \mathrm{hr})$ & $64.0(70.0)$ & $44.5(62.0)$ & 0.001 \\
\hline CRP (mg/L) & $15.9(92.2)$ & $2.3(13.8)$ & $<0.001$ \\
\hline \multicolumn{4}{|l|}{ During the follow-up period } \\
\hline Follow-up duration (months) & $32.0(59.5)$ & $36.2(64.8)$ & 0.347 \\
\hline
\end{tabular}

Values are expressed as a median (interquartile range, IQR) or $\mathrm{N}(\%)$.

AAV: antineutrophil cytoplasmic antibody (ANCA)-associated vasculitis; MetS: metabolic syndrome; NCEPATP-III: national cholesterol education program-adult treatment panel III; HDL: high-density lipoprotein; BMI: body mass index; MPA: microscopic polyangiitis; GPA: granulomatosis with polyangiitis; EGPA: eosinophilic granulomatosis with polyangiitis; MPO: myeloperoxidase; P: perinuclear; PR3: proteinase 3; C: cytoplasmic; BVAS: Birmingham vasculitis activity score; FFS: five-factor score; ESR: erythrocyte sedimentation rate; CRP: C-reactive protein; ESRD: end-stage renal disease; CVA: cerebrovascular accident; CVD: cardiovascular disease. 


\begin{tabular}{|c|c|c|c|}
\hline AAV patients & $\begin{array}{l}\text { AAV patients } \\
\text { with MetS ( }= \\
\text { 88) }\end{array}$ & $\begin{array}{l}\text { AAV patients } \\
\text { without Mets ( } \mathrm{N}= \\
\text { 85) }\end{array}$ & $\begin{array}{l}\mathrm{P}- \\
\text { value }\end{array}$ \\
\hline \multicolumn{4}{|l|}{ Poor outcomes } \\
\hline All-cause mortality (N, (\%)) & $8(9.1)$ & $6(7.1)$ & 0.624 \\
\hline Follow-up duration for death (months) & $32.0(59.5)$ & $36.2(64.8)$ & 0.359 \\
\hline Relapse (N, (\%)) & $31(35.2)$ & $25(29.4)$ & 0.414 \\
\hline Follow-up duration for relapse (months) & $20.1(41.9)$ & $17.8(37.8)$ & 0.524 \\
\hline $\operatorname{ESRD}(\mathrm{N},(\%))$ & $22(25.0)$ & $11(12.9)$ & 0.044 \\
\hline Follow-up duration for ESRD (months) & $20.2(55.5)$ & $23.6(62.9)$ & 0.616 \\
\hline CVA $(\mathrm{N},(\%))$ & $8(9.1)$ & $4(4.7)$ & 0.371 \\
\hline Follow-up duration for CVA (months) & $28.0(62.9)$ & $31.5(59.5)$ & 0.522 \\
\hline CVD $(\mathrm{N},(\%))$ & $11(12.5)$ & $1(1.2)$ & 0.003 \\
\hline Follow-up duration for CVD (months) & $31.1(60.4)$ & $36.2(64.8)$ & 0.935 \\
\hline \multicolumn{4}{|l|}{ Medication administered (N, (\%)) } \\
\hline Glucocorticoid & $83(94.3)$ & $79(92.9)$ & 0.711 \\
\hline Cyclophosphamide & $45(51.1)$ & $41(48.2)$ & 0.703 \\
\hline Rituximab & $15(17.0)$ & $11(12.9)$ & 0.450 \\
\hline Azathioprine & $45(51.1)$ & $38(44.7)$ & 0.397 \\
\hline Mycophenolate mofetil & $11(12.5)$ & $11(12.9)$ & 0.931 \\
\hline Tacrolimus & $3(3.4)$ & $7(8.2)$ & 0.206 \\
\hline Methotrexate & $5(5.7)$ & $12(14.1)$ & 0.062 \\
\hline \multicolumn{4}{|c|}{ Values are expressed as a median (interquartile range, IQR) or $\mathrm{N}(\%)$. } \\
\hline \multicolumn{4}{|c|}{$\begin{array}{l}\text { AAV: antineutrophil cytoplasmic antibody (ANCA)-associated vasculitis; MetS: metabolic syndrome; NCEP- } \\
\text { ATP-III: national cholesterol education program-adult treatment panel III; HDL: high-density lipoprotein; BMI: } \\
\text { body mass index; MPA: microscopic polyangiitis; GPA: granulomatosis with polyangiitis; EGPA: eosinophilic } \\
\text { granulomatosis with polyangiitis; MPO: myeloperoxidase; P: perinuclear; PR3: proteinase 3; C: cytoplasmic; } \\
\text { BVAS: Birmingham vasculitis activity score; FFS: five-factor score; ESR: erythrocyte sedimentation rate; CRP: } \\
\text { C-reactive protein; ESRD: end-stage renal disease; CVA: cerebrovascular accident; CVD: cardiovascular } \\
\text { disease. }\end{array}$} \\
\hline
\end{tabular}

\section{Multivariable logistic regression analysis of variables at diagnosis for the cross-sectional MetS in AAV patients}

We categorised variables at diagnosis with statistical significance in the comparison analysis into the two groups: the conventional risk factors for MetS and AAV-specific inflammatory indices as shown in Supplementary Table 1. To determine the independent predictor of the cross-sectional MetS at diagnosis, we 
conducted the multivariable logistic regression analysis and found that BMI (OR 1.481), diabetes mellitus (OR 7.629), hypertension (OR 16.054) and dyslipidaemia (OR 8.800) were significantly associated with the crosssectional MetS. Whereas, none of the AAV-specific inflammatory indices was associated with the cross-sectional MetS.

\section{Comparison of the effect of MetS at diagnosis on the risk of the outcomes of AAV during follow-up}

We simply compared the frequencies of the poor outcomes of AAV between the two groups and found that ESRD and CVD occurred in AAV patients with MetS more frequently than those without MetS during follow-up (Table 3). We also compared the cumulative risk of each poor-outcome during the follow-up period based on each poor outcome occurrence between the two groups using the Kaplan-Meier survival analysis. AAV patients with MetS exhibited the lower cumulative CVD-free survival rate than those without MetS during the follow-up period based on CVD occurrence (Fig. 2). Furthermore, we assessed the effect of MetS at diagnosis on the risk of CVD by dividing AAV patients into two categories based on BMI of $25 \mathrm{~kg} / \mathrm{m}^{2}$. Among nonobese AAV patients, MetS at diagnosis significantly reduced the cumulative CVD-free survival rate. However, among obese AAV patients, there was no significant difference in the cumulative CVD-free survival rate between AAV patients with and without MetS ( $F_{I G}$ 2).

\section{Hazard ratio of variables at diagnosis for the risk of CVD during follow-up in 173 AAV patients}

Firstly, in cases of all AAV patients, in the univariable Cox hazards model analysis, male gender, BVAS, FFS, diabetes mellitus, dyslipidaemia, fasting glucose level and MetS at diagnosis were significantly associated with CVD occurrence during follow-up. In the multivariable Cox hazards model analysis of variables with significance in the univariable analysis, only male gender (HR 6.006, 95\% confidence interval (CI) 1.486, 24.283) was significantly associated with CVD occurrence during follow-up. With the same assumption above, among male gender, BVAS, FFS and MetS at diagnosis, both male gender (HR 4.625, 95\% Cl 1.258, 16.996) and MetS at diagnosis (HR 9.864, 95\% Cl 1.136, 85.679) were significantly associated with CVD during follow-up (Supplementary Table 2).

\section{Hazard ratio of variables at diagnosis for the risk of CVD during follow-up in 144 nonobese AAV patients}

In cases of nonobese AAV patients, in the univariable Cox hazards model analysis, BVAS, dyslipidaemia, haemoglobin, fasting glucose and MetS at diagnosis were significantly associated with CVD during follow-up. In the multivariable analysis, only BVAS at diagnosis (HR 1.157, 95CI 1.038, 1.289) was significantly associated with CVD during follow-up. Given that diabetes, dyslipidaemia and fasting glucose are closely related to components of the 2005 NCEP-ATP-III criteria, they could be deleted in the multivariable analysis in order to clarify the effect of variables at diagnosis on the risk of CVD. Thus, only BVAS, haemoglobin, and MetS at diagnosis were included in the multivariable analysis, in which, both BVAS (HR 1.159, 95\% Cl 1.039, 1.293) and MetS at diagnosis (HR 9.036, 95\% Cl 1.011, 80.786) were significantly associated with CVD during follow-up (Table 4). 
Table 4

Univariable and multivariable Cox hazards model analyses of variables at diagnosis for CVD occurrence during follow-up in AAV patients with normal BMI $(B M I<25 \mathrm{~kg} / \mathrm{m} 2)(\mathrm{N}=145)$

\begin{tabular}{|c|c|c|c|c|c|c|c|c|c|}
\hline \multirow[t]{2}{*}{ Variables } & \multicolumn{3}{|c|}{ Univariable } & \multicolumn{3}{|c|}{ Multivariable* } & \multicolumn{3}{|c|}{ Multivariable } \\
\hline & HR & $95 \% \mathrm{Cl}$ & $\begin{array}{l}P \\
\text { value }\end{array}$ & HR & $95 \% \mathrm{Cl}$ & $\begin{array}{l}P \\
\text { value }\end{array}$ & HR & $95 \% \mathrm{Cl}$ & $\begin{array}{l}P \\
\text { value }\end{array}$ \\
\hline \multicolumn{10}{|l|}{$\begin{array}{l}\text { Demographic } \\
\text { data }\end{array}$} \\
\hline Age & 1.007 & $\begin{array}{l}0.962 \\
1.055\end{array}$ & 0.757 & & & & & & \\
\hline Male gender & 3.543 & $\begin{array}{l}0.864 \\
14.537\end{array}$ & 0.079 & & & & & & \\
\hline BMI & 1.263 & $\begin{array}{l}0.908 \\
1.758\end{array}$ & 0.165 & & & & & & \\
\hline \multicolumn{10}{|l|}{$\begin{array}{l}\text { ANCA } \\
\text { positivity }\end{array}$} \\
\hline $\begin{array}{l}\text { MPO-ANCA } \\
\text { (or P-ANCA) } \\
\text { positivity }\end{array}$ & 1.680 & $\begin{array}{l}0.387 \\
7.292\end{array}$ & 0.489 & & & & & & \\
\hline $\begin{array}{l}\text { PR3-ANCA (or } \\
\text { C-ANCA) } \\
\text { positivity }\end{array}$ & 0.565 & $\begin{array}{l}0.069 \\
4.600\end{array}$ & 0.565 & & & & & & \\
\hline $\begin{array}{l}\text { ANCA } \\
\text { positivity }\end{array}$ & 1.781 & $\begin{array}{l}0.306 \\
10.362\end{array}$ & 0.521 & & & & & & \\
\hline \multicolumn{10}{|l|}{$\begin{array}{l}\text { AAV-specific } \\
\text { indices }\end{array}$} \\
\hline BVAS & 1.167 & $\begin{array}{l}1.063 \\
1.281\end{array}$ & 0.001 & 1.157 & $\begin{array}{l}1.038 \\
1.289\end{array}$ & 0.008 & 1.159 & $\begin{array}{l}1.039 \\
1.293\end{array}$ & 0.008 \\
\hline FFS & 1.697 & $\begin{array}{l}0.977 \\
2.947\end{array}$ & 0.061 & & & & & & \\
\hline $\begin{array}{l}\text { Comorbidities } \\
(\mathrm{N},(\%))\end{array}$ & & & & & & & & & \\
\hline
\end{tabular}

*: Dyslipidaemia and fasting glucose, which exhibited statistical significance in the univariable analysis, were included in the multivariable analysis.

**: Dyslipidaemia and fasting glucose, which exhibited statistical significance in the univariable analysis, were excluded from the multivariable analysis because they are related to five components of the 2005 NCEP-ATP-III criteria for MetS.

CVD: cardiovascular disease; AAV: antineutrophil cytoplasmic autoantibody (ANCA)-associated vasculitis; HR: hazard ratio, Cl: confidence interval; BMI: body mass index; MPO: myeloperoxidase; P: perinuclear; PR3: proteinase 3; C: cytoplasmic; BVAS: Birmingham vasculitis activity score; FFS: five-factor score; ESR: erythrocyte sedimentation rate; CRP: C-reactive protein; MetS: metabolic syndrome; NCEP-ATP-III: national cholesterol education program-adult treatment panel III. 


\begin{tabular}{|c|c|c|c|c|c|c|c|c|c|}
\hline \multirow[t]{2}{*}{ Variables } & \multicolumn{3}{|c|}{ Univariable } & \multicolumn{3}{|c|}{ Multivariable $^{*}$} & \multicolumn{3}{|c|}{ Multivariable } \\
\hline & HR & $95 \% \mathrm{Cl}$ & $\begin{array}{l}P \\
\text { value }\end{array}$ & HR & $95 \% \mathrm{Cl}$ & $\begin{array}{l}P \\
\text { value }\end{array}$ & HR & $95 \% \mathrm{Cl}$ & $\begin{array}{l}P \\
\text { value }\end{array}$ \\
\hline $\begin{array}{l}\text { Chronic } \\
\text { kidney } \\
\text { disease } \\
\text { (stage 3-5) }\end{array}$ & 0.719 & $\begin{array}{l}0.148 \\
3.485\end{array}$ & 0.682 & & & & & & \\
\hline $\begin{array}{l}\text { Diabetes } \\
\text { mellitus }\end{array}$ & 3.314 & $\begin{array}{l}0.828 \\
13.271\end{array}$ & 0.091 & & & & & & \\
\hline Hypertension & 3.432 & $\begin{array}{l}0.70 \\
16.834\end{array}$ & 0.129 & & & & & & \\
\hline Dyslipidaemia & 5.869 & $\begin{array}{l}1.456 \\
23.659\end{array}$ & 0.013 & 2.045 & $\begin{array}{l}0.415 \\
10.069\end{array}$ & 0.379 & & & \\
\hline $\begin{array}{l}\text { Interstitial } \\
\text { lung disease }\end{array}$ & 0.489 & $\begin{array}{l}0.061 \\
3.927\end{array}$ & 0.501 & & & & & & \\
\hline \multicolumn{10}{|l|}{$\begin{array}{l}\text { Laboratory } \\
\text { results }\end{array}$} \\
\hline $\begin{array}{l}\text { White blood } \\
\text { cell count } \\
\left(/ \mathrm{mm}^{3}\right)\end{array}$ & 1.000 & $\begin{array}{l}1.000 \\
1.000\end{array}$ & 0.191 & & & & & & \\
\hline $\begin{array}{l}\text { Haemoglobin } \\
(\mathrm{g} / \mathrm{dL})\end{array}$ & 0.697 & $\begin{array}{l}0.487 \\
0.997\end{array}$ & 0.048 & 0.881 & $\begin{array}{l}0.635 \\
1.223\end{array}$ & 0.449 & 0.922 & $\begin{array}{l}0.657 \\
1.295\end{array}$ & 0.640 \\
\hline $\begin{array}{l}\text { Platelet count } \\
(\times\end{array}$ & 1.001 & $\begin{array}{l}0.997 \\
1.005\end{array}$ & 0.717 & & & & & & \\
\hline $\begin{array}{l}\text { Fasting } \\
\text { glucose } \\
(\mathrm{mg} / \mathrm{dL})\end{array}$ & 1.014 & $\begin{array}{l}1.003 \\
1.026\end{array}$ & 0.011 & 1.013 & $\begin{array}{l}1.000 \\
1.027\end{array}$ & 0.057 & & & \\
\hline $\begin{array}{l}\text { Creatinine } \\
(\mathrm{mg} / \mathrm{dL})\end{array}$ & 1.234 & $\begin{array}{l}0.957 \\
1.593\end{array}$ & 0.106 & & & & & & \\
\hline $\begin{array}{l}\text { Serum } \\
\text { albumin } \\
\text { (g/dL) }\end{array}$ & 0.453 & $\begin{array}{l}0.178 \\
1.150\end{array}$ & 0.096 & & & & & & \\
\hline
\end{tabular}

*: Dyslipidaemia and fasting glucose, which exhibited statistical significance in the univariable analysis, were included in the multivariable analysis.

**: Dyslipidaemia and fasting glucose, which exhibited statistical significance in the univariable analysis, were excluded from the multivariable analysis because they are related to five components of the 2005 NCEP-ATP-III criteria for MetS.

CVD: cardiovascular disease; AAV: antineutrophil cytoplasmic autoantibody (ANCA)-associated vasculitis; HR: hazard ratio, Cl: confidence interval; BMI: body mass index; MPO: myeloperoxidase; P: perinuclear; PR3: proteinase 3; C: cytoplasmic; BVAS: Birmingham vasculitis activity score; FFS: five-factor score; ESR: erythrocyte sedimentation rate; CRP: C-reactive protein; MetS: metabolic syndrome; NCEP-ATP-III: national cholesterol education program-adult treatment panel III. 


\begin{tabular}{|c|c|c|c|c|c|c|c|c|c|}
\hline \multirow[t]{2}{*}{ Variables } & \multicolumn{3}{|c|}{ Univariable } & \multicolumn{3}{|c|}{ Multivariable ${ }^{*}$} & \multicolumn{3}{|c|}{ Multivariable } \\
\hline & HR & $95 \% \mathrm{Cl}$ & $\begin{array}{l}\mathrm{P} \\
\text { value }\end{array}$ & HR & $95 \% \mathrm{Cl}$ & $\begin{array}{l}P \\
\text { value }\end{array}$ & HR & $95 \% \mathrm{Cl}$ & $\begin{array}{l}P \\
\text { value }\end{array}$ \\
\hline $\mathrm{ESR}(\mathrm{mm} / \mathrm{hr})$ & 1.010 & $\begin{array}{l}0.993 \\
1.026\end{array}$ & 0.244 & & & & & & \\
\hline CRP (mg/L) & 1.007 & $\begin{array}{l}0.998, \\
1.015\end{array}$ & 0.114 & & & & & & \\
\hline $\begin{array}{l}\text { Total } \\
\text { cholesterol } \\
(\mathrm{mg} / \mathrm{dL})\end{array}$ & 1.001 & $\begin{array}{l}0.986 \\
1.016\end{array}$ & 0.935 & & & & & & \\
\hline $\begin{array}{l}\text { Presence of } \\
\text { MetS }\end{array}$ & 10.029 & $\begin{array}{l}1.249 \\
80.541\end{array}$ & 0.030 & 5.107 & $\begin{array}{l}0.428 \\
60.916\end{array}$ & 0.197 & 9.036 & $\begin{array}{l}1.011, \\
80.786\end{array}$ & 0.049 \\
\hline \multicolumn{10}{|c|}{$\begin{array}{l}\text { *: Dyslipidaemia and fasting glucose, which exhibited statistical significance in the univariable analysis, were } \\
\text { included in the multivariable analysis. }\end{array}$} \\
\hline \multicolumn{10}{|c|}{$\begin{array}{l}\text { **: Dyslipidaemia and fasting glucose, which exhibited statistical significance in the univariable analysis, } \\
\text { were excluded from the multivariable analysis because they are related to five components of the } 2005 \\
\text { NCEP-ATP-III criteria for MetS. }\end{array}$} \\
\hline \multicolumn{10}{|c|}{$\begin{array}{l}\text { CVD: cardiovascular disease; AAV: antineutrophil cytoplasmic autoantibody (ANCA)-associated vasculitis; } \\
\text { HR: hazard ratio, Cl: confidence interval; BMI: body mass index; MPO: myeloperoxidase; P: perinuclear; PR3: } \\
\text { proteinase 3; C: cytoplasmic; BVAS: Birmingham vasculitis activity score; FFS: five-factor score; ESR: } \\
\text { erythrocyte sedimentation rate; CRP: C-reactive protein; MetS: metabolic syndrome; NCEP-ATP-III: national } \\
\text { cholesterol education program-adult treatment panel III. }\end{array}$} \\
\hline
\end{tabular}

\section{Discussion}

In this study, we assessed the effect of variables at diagnosis on the risk of CVD during follow-up in AAV patients and found several interesting findings. Firstly, the prevalence of MetS based on the 2005 NCEP-ATP-III criteria was $50.9 \%$ in all AAV patients, which was significantly higher than $37.8 \%$ in age- and gender-matched controls. The 2013 annual report regarding the prevalence of MetS in approximately 10 million Korean individuals with an average age of 50.8 years and BMI of $23.9 \mathrm{~kg} / \mathrm{m}^{2}$ analysed the overall prevalence of MetS as $30.5 \%$ [24]. The next version of the report, Metabolic Syndrome Fact Sheet in Korea 2018, reported the increased prevalence of Mets of Korean people of an average age of $50 \mathrm{~s}$ up to $37.9 \%$ [25], which supports that controls in this study were representative of the general Korean population of an average age of $50 \mathrm{~s}$. Moreover, the prevalence of MetS in AAV patients in Korea was slightly higher than that in the UK [12]. Despite insufficient studies investigating the prevalence of MetS in AAV patients, this discordance might be considered to appear due to the different ethnic or geographical backgrounds [26].

Secondly, the prevalence of MetS was significantly higher in nonobese AAV patients than that in nonobese controls ( $46.5 \%$ vs. $28.2 \%)$. This result may suggest the contribution of the inflammatory burden of AAV to the presence of MetS in AAV patients beyond obesity and its related complications. Interestingly, in the UK study, no difference in BMI between AAV patients and controls was observed [12]. Whereas, in our study, BMI of AAV patients was significantly lower than that of controls, which exhibited an opposite tendency of the prevalence of MetS. Although BMI is not one of the components of the 2005 NCEP-ATP-III criteria, BMI is another independent 
index for determining obesity and considered one of the risks for MetS [6, 27]. This inverse tendency suggests that another unique risk factor exists in AAV patients other than the conventional risk factors for MetS in normal people and it was assumed as the inflammatory burden of AAV. To prove this assumption, we tried to compare the cross-sectional BVAS or FFS between the two studies but unfortunately, we could not due to no information on BVAS in the UK study.

Thirdly, unlike the comparison analysis between AAV patients and controls, BMI was strongly associated with the cross-sectional MetS as shown in the comparison analysis between AAV patients with MetS and those without MetS. Based on this result, it might be assumed that the general association between obesity and MetS became apparent when compared only in AAV patients, resulting from minimizing the influence of the inflammatory burden of AAV. However, the burden of inflammation was not thoroughly removed, because BVAS was assessed significantly elevated in AAV patients with MetS, compared to those without MetS. Thus, this result may suggest the cooperative contribution of AAV activity to the presence of MetS in AAV patients along with obesity and its related complications.

Supposed that variables directly related to the 2005 NCEP-ATP-III criteria were excluded, two categories of the risk factors at diagnosis for the cross-section MetS could be organized: one is the conventional risk factors such as age, BMI, diabetes mellitus, hypertension and dyslipidaemia; and the other is the AAV-specific inflammatory variables such as BVAS, FFS, haemoglobin, platelet count, creatinine, serum albumin, ESR and CRP at diagnosis. Using these variables, we conducted the multivariable logistic regression analysis and found that BMI, diabetes mellitus, hypertension and dyslipidaemia were independently and significantly associated with the crosssectional MetS at diagnosis. By contrast, none of the AAV-specific inflammatory variables were significantly associated with the cross-sectional MetS. This analysis gave two conclusions: one is that BVAS itself might not be independently associated with the cross-sectional MetS in AAV patients, and the other is that BMI might independently contribute to the cross-sectional MetS in AAV patients and AAV activity might consolidate the association between BMI and MetS at diagnosis.

Since BMI was an independent variable that could predict the cross-sectional MetS in AAV patients, we calculated the optimal cut-off of BMI at diagnosis for the cross-sectional Mets using the ROC curve analysis. We determined the BMI of $22.9 \mathrm{~kg} / \mathrm{m}^{2}$ as the cut-off for a strong predictor of the cross-sectional MetS (area 0.686 , $95 \% \mathrm{Cl} 0.606,0.766, \mathrm{P}<0.001$, sensitivity $62.5 \%$, specificity $75.3 \%$ ) (Supplementary Fig. $1 \mathrm{~A}$ ). When we classified AAV patients into the two groups based on the cut-off of BMI and assessed its relative risk for the occurrence of the cross-sectional MetS using the chi-square test, $76 \mathrm{AAV}$ patients were partitioned into the group of $\mathrm{BMI} \geq$ $22.9 \mathrm{~kg} / \mathrm{m}^{2}$. The cross-sectional MetS was identified more frequently in AAV patients with $\mathrm{BMI} \geq 22.9 \mathrm{~kg} / \mathrm{m}^{2}$ than those without $(72.4 \%$ vs. $34.0 \%, \mathrm{P}<0.001)$. Furthermore, patients with $\mathrm{BMI} \geq 22.9 \mathrm{~kg} / \mathrm{m}^{2}$ had the significantly higher relative risk of having the cross-sectional MetS than those without (RR $5.079,95 \% \mathrm{Cl} 2.638,9.780$ ) (Supplementary Fig. 1B).

Prior to the investigation, it should be noted that except for relapse of AAV, Mets could increase the risks of allcause mortality [28], chronic kidney disease or ESRD [29], CVA [30] and CVD [28, 31, 32] in both AAV patients and the general population with MetS. In addition, AAV itself without MetS also could increase the risk for CVD compared to healthy people $[14,33]$. Therefore, it should not be ignored that both AAV entity and the crosssectional MetS at diagnosis may be simultaneously engaged in CVD occurrence in AAV patients: MetS might 
significantly initiate CVD occurrence and AAV might accelerate it. On the other hand, unlike, the UK study [12], we could not find any link between MetS at diagnosis and relapse during follow-up in this study.

Fourthly, Mets at diagnosis significantly reduced the cumulative CVD-free survival rate and both BVAS and Mets at diagnosis significantly associated with CVD in nonobese AAV patients. In the survival analysis, MetS at diagnosis significantly reduced the cumulative CVD-free survival rate only in nonobese AAV patients. Whereas, obese AAV patients showed no association between MetS at diagnosis and CVD occurrence during follow-up. This result may suggest that the independent contribution of MetS to the development of CVD would have been offset because MetS is closely related to obesity itself and obesity-related complications in obese AAV patients. For this reason, the effect of MetS on the risk of CVD might be clearer in nonobese AAV patients. In addition, to discover the independent predictors of and contributors to CVD occurrence during follow-up in nonobese AAV patients, we conducted the multivariable Cox hazards model analysis using variables with $P$ value less than 0.05 in the univariable analysis. In the multivariable analysis excluding variables related to the 2005 NCEP-ATP-III criteria, BVAS and MetS at diagnosis had influence on CVD in nonobese AAV patients. This result might support our assumption that both AAV entity and metabolic abnormalities could enhance the risk of CVD in nonobese AAV patients.

Our study has several limitations. Controls, who visited Severance Executive Healthcare Clinic in Severance Hospital, and the two-thirds of AAV patients, who belong to the prospective cohort of AAV in our hospital, had information on smoking history, alcohol consumption, and family history of MetS and CVD. However, we could not gather them from all AAV patients and controls due to the nature of a retrospective study. In addition, the number of AAV patients of this study, particularly patients with CVD occurrence, was not large enough to represent all Korean patients with AAV, due to a limitation of a monocentric study. Nevertheless, we believe that this study has significant clinical implications as a pilot study in that we clarified the effect of BVAS and MetS at diagnosis on the risk of CVD in all or nonobese AAV patients, for the first time. In the near future, a prospective and multicentre study with a larger number of AAV patients will compensate for the limitations of this study.

\section{Conclusions}

The prevalence of MetS at diagnosis was $50.9 \%$ in all AAV patients and $46.5 \%$ in nonobese AAV patients, both of which were significantly higher than those in all controls and nonobese controls. Furthermore, both BVAS and MetS at diagnosis increased the risk of CVD in nonobese AAV patients.

\section{Declarations}

\section{Ethics Approval and consent to participate}

This study was approved by the Institutional Review Board (IRB) of Severance Hospital (4-2017-0673 for AAV patients and 4-2018-0856 for controls), who waived the need for the written informed consent, as this was a retrospective study.

\section{Consent for publication}

Not applicable 
Availability of data and material

Not applicable

\section{Competing interests}

The authors declare no competing interests

\section{Funding}

This research was supported by Basic Science Research Program through the National Research Foundation of Korea (NRF) funded by the Ministry of Education (2017R1D1A1B03029050) and a grant from the Korea Health Technology R\&D Project through the Korea Health Industry Development Institute, funded by the Ministry of Health and Welfare, Republic of Korea (HI14C1324).

\section{Author's contributions}

All authors contributed to the study concept, design, acquisition and interpretation of data. SBL, HCK, JYP and SWL performed the statistical analysis. SBL, HCK and SWL drafted the manuscript. All authors revised and approved the manuscript for publication.

\section{Abbreviations}

AAV: ANCA-associated vasculitis; ANCA: antineutrophil cytoplasmic antibody; BMI: body mass index; BVAS: Birmingham vasculitis activity score; C: cytoplasmic; $\mathrm{Cl}$ : confidence interval; CVA: cerebrovascular accident; CVD: cardiovascular disease; DUR: Drug Utilization Review; EGPA: eosinophilic granulomatosis with polyangiitis; ESRD: end-stage renal disease; EULAR: European League Against Rheumatism; FFS: five-factor score; GPA: granulomatosis with polyangiitis; HDL: high-density lipoprotein; HR: hazard ratios; ICD: International Classification Diseases; IL: interleukin; IRB: Institutional Review Board; MetS: metabolic syndrome; MPA: microscopic polyangiitis; MPO: myeloperoxidase; NCEP-ATP-III: National Cholesterol Education Program Adults Treatment Panel III; OR: odds ratio; P: perinuclear; PR3: proteinase 3; ROC: receiver operator characteristic; RR: relative risk; TNF: tumour necrosis factor; WHO: World Health Organization.

\section{References}

1. Eckel RH, Grundy SM, Zimmet PZ. The metabolic syndrome. Lancet. 2005;365:1415-28.

2. Miranda PJ, DeFronzo RA, Califf RM, Guyton JR. Metabolic syndrome: definition, pathophysiology, and mechanisms. Am Heart J. 2005;149:33-45.

3. Alberti KG, Zimmet PZ. Definition, diagnosis and classification of diabetes mellitus and its complications. Part 1: diagnosis and classification of diabetes mellitus provisional report of a WHO consultation. Diabet Med. 1998;15:539-53.

4. Balkau B, Charles MA. Comment on the provisional report from the WHO consultation. European Group for the Study of Insulin Resistance (EGIR). Diabet Med. 1999;16:442-3.

5. Expert Panel on Detection. Evaluation, and Treatment of High Blood Cholesterol in Adults. Executive Summary of The Third Report of The National Cholesterol Education Program (NCEP) Expert Panel on 
Detection, Evaluation, And Treatment of High Blood Cholesterol In Adults (Adult Treatment Panel III). JAMA. 2001;285:2486-97.

6. National Heart, Lung, and Blood Institute; American Heart Association

Grundy SM, Brewer HB Jr, Cleeman JI, Smith SC Jr, Lenfant C. National Heart, Lung, and Blood Institute;

American Heart Association: Definition of metabolic syndrome: report of the National Heart, Lung, and Blood Institute/American Heart Association conference on scientific issues related to definition. Arterioscler Thromb Vasc Biol 2004, 24: e13-e18.

7. Srikanthan K, Feyh A, Visweshwar H, Shapiro JI, Sodhi K. Systematic Review of Metabolic Syndrome Biomarkers: A Panel for Early Detection, Management, and Risk Stratification in the West Virginian Population. Int J Med Sci. 2016;13:25-38.

8. Zmora N, Bashiardes S, Levy M, Elinav E. The Role of the Immune System in Metabolic Health and Disease. Cell Metab. 2017;25:506-21.

9. Medina G, Vera-Lastra O, Peralta-Amaro AL, Jiménez-Arellano MP, Saavedra MA, Cruz-Domínguez MP, Jara LJ. Metabolic syndrome, autoimmunity and rheumatic diseases. Pharmacol Res. 2018;133:277-88.

10. Jennette JC, Falk RJ, Bacon PA, Basu N, Cid MC, Ferrario F, Flores-Suarez LF, Gross WL, Guillevin L, Hagen EC, Hoffman GS, Jayne DR, Kallenberg CG, Lamprecht P, Langford CA, Luqmani RA, Mahr AD, Matteson EL, Merkel PA, Ozen S, Pusey CD, Rasmussen N, Rees AJ, Scott DG, Specks U, Stone JH, Takahashi K, Watts RA: 2012 revised International Chapel Hill Consensus Conference Nomenclature of Vasculitides. Arthritis Rheum 2013, 65: 1-11.

11. Watts R, Lane S, Hanslik T, Hauser T, Hellmich B, Koldingsnes W, Mahr A, Segelmark M, Cohen-Tervaert JW, Scott D. Development and validation of a consensus methodology for the classification of the ANCAassociated vasculitides and polyarteritis nodosa for epidemiological studies. Ann Rheum Dis. 2007;66:2227.

12. Petermann Smits DR, Wilde B, Kianersi Adegani M, de Jongh H, van Paassen P, Cohen Tervaert JW. Metabolic syndrome in ANCA-associated vasculitis. Rheumatology. 2013;52:197-203.

13. Morgan MD, Turnbull J, Selamet U, Kaur-Hayer M, Nightingale P, Ferro CJ, Savage CO, Harper L. Increased incidence of cardiovascular events in patients with antineutrophil cytoplasmic antibody-associated vasculitides: a matched-pair cohort study. Arthritis Rheum. 2009;60:3493-500.

14. Houben E, Penne EL, Voskuyl AE, van der Heijden JW, Otten RHJ, Boers M, Hoekstra T. Cardiovascular events in anti-neutrophil cytoplasmic antibody-associated vasculitis: a meta-analysis of observational studies. Rheumatology. 2018;57:555-62.

15. Yates M, Watts RA, Bajema IM, Cid MC, Crestani B, Hauser T, Hellmich B, Holle JU, Laudien M, Little MA, Luqmani RA, Mahr A, Merkel PA, Mills J, Mooney J, Segelmark M, Tesar V, Westman K, Vaglio A, Yalçındağ N, Jayne DR, Mukhtyar C. EULAR/ERA-EDTA recommendations for the management of ANCA-associated vasculitis. Ann Rheum Dis. 2016;75:1583-94.

16. Voulgari C, Tentolouris N, Dilaveris P, Tousoulis D, Katsilambros N, Stefanadis C. Increased heart failure risk in normal-weight people with metabolic syndrome compared with metabolically healthy obese individuals. J Am Coll Cardiol. 2011;58:1343-50.

17. Kim SY, Kim MS, Sim S, Park B, Choi HG. Association Between Obesity and Falls Among Korean Adults: A Population-Based Cross-Sectional Study. Med (Baltim). 2016;95:e3130. 
18. Mukhtyar C, Lee R, Brown D, Carruthers D, Dasgupta B, Dubey S, Flossmann O, Hall C, Hollywood J, Jayne D, Jones R, Lanyon P, Muir A, Scott D, Young L, Luqmani RA. Modification and validation of the Birmingham Vasculitis Activity Score (version 3). Ann Rheum Dis. 2009;68:1827-32.

19. Guillevin L, Pagnoux C, Seror R, Mahr A, Mouthon L, Le Toumelin P, French Vasculitis Study Group (FVSG). The Five-Factor Score revisited: assessment of prognoses of systemic necrotizing vasculitides based on the French Vasculitis Study Group (FVSG) cohort. Med (Baltim). 2011;90:19-27.

20. Sung MJ, Lim TS, Jeon MY, Lee HW, Kim BK, Kim DY, Ahn SH, Han KH, Park JY, Kim SU. Sarcopenia Is Independently Associated with the Degree of Liver Fibrosis in Patients with Type 2 Diabetes Mellitus. Gut Liver 2020 Mar 6. doi:10.5009/gnl19126. [Epub ahead of print].

21. Kwon HS, Park YM, Lee HJ, Lee JH, Choi YH, Ko SH, Lee JM, Kim SR, Kang SY, Lee WC, Ahn MS, Noh JH, Kang JM, Kim DS, Yoon KH, Cha BY, Lee KW, Kang SK, Son HY. Prevalence and clinical characteristics of the metabolic syndrome in middle-aged Korean adults. Korean J Intern Med. 2005;20:310-6.

22. Csernok E, Moosig F. Current and emerging techniques for ANCA detection in vasculitis. Nat Rev Rheumatol. 2014;10:494-501.

23. Bossuyt X, Cohen Tervaert JW, Arimura Y, Blockmans D, Flores-Suárez LF, Guillevin L, Hellmich B, Jayne D, Jennette JC, Kallenberg CGM, Moiseev S, Novikov P, Radice A, Savige JA, Sinico RA, Specks U, van Paassen P, Zhao MH, Rasmussen N, Damoiseaux J, Csernok E. Position paper: Revised 2017 international consensus on testing of ANCAs in granulomatosis with polyangiitis and microscopic polyangiitis. Nat Rev Rheumatol. 2017;13:683-92.

24. Lee SE, Han K, Kang YM, Kim SO, Cho YK, Ko KS, Park JY, Lee KU, Koh EH, Taskforce Team of Diabetes Fact Sheet of the Korean Diabetes Association. Trends in the prevalence of metabolic syndrome and its components in South Korea: Findings from the Korean National Health Insurance Service Database (20092013). PLoS One. 2018;13:e0194490.

25. Metabolic Syndrome Fact Sheet in Korea. 2018, http://kscms.org/en/uploads/Metabolic_Syndrome.pdf.

26. Alberti KG, Zimmet P, Shaw J, IDF Epidemiology Task Force Consensus Group. The metabolic syndrome-a new worldwide definition. Lancet. 2005;366:1059-62.

27. Kobo O, Leiba R, Avizohar O, Karban A. Normal body mass index (BMI) can rule out metabolic syndrome: An Israeli cohort study. Med (Baltim). 2019;98:e14712.

28. Ju SY, Lee JY, Kim DH. Association of metabolic syndrome and its components with all-cause and cardiovascular mortality in the elderly: A meta-analysis of prospective cohort studies. Med (Baltim). 2017;96:e8491.

29. Huh JH, Yadav D, Kim JS, Son JW, Choi E, Kim SH, Shin C, Sung KC, Kim JY. An association of metabolic syndrome and chronic kidney disease from a 10-year prospective cohort study. Metabolism. 2017;67:54-61.

30. Jabir NR, Firoz CK, Khan MS, Zaidi SK, Ashraf GM, Shakil S, Kamal MA, Tabrez S. Potential Linkage Between Cerebrovascular Diseases and Metabolic Syndrome. Curr Drug Metab. 2017;18:62-8.

31. Mottillo S, Filion KB, Genest J, Joseph L, Pilote L, Poirier P, Rinfret S, Schiffrin EL, Eisenberg MJ. The metabolic syndrome and cardiovascular risk a systematic review and meta-analysis. J Am Coll Cardiol. 2010;56:1113-32.

32. Galassi A, Reynolds K, He J. Metabolic syndrome and risk of cardiovascular disease: a meta-analysis. Am J Med. 2006;119:812-9. 
33. Berti A, Matteson EL, Crowson CS, Specks U, Cornec D. Risk of Cardiovascular Disease and Venous Thromboembolism Among Patients With Incident ANCA-Associated Vasculitis: A 20-Year Population-Based Cohort Study. Mayo Clin Proc. 2018;93:597-606.

\section{Figures}
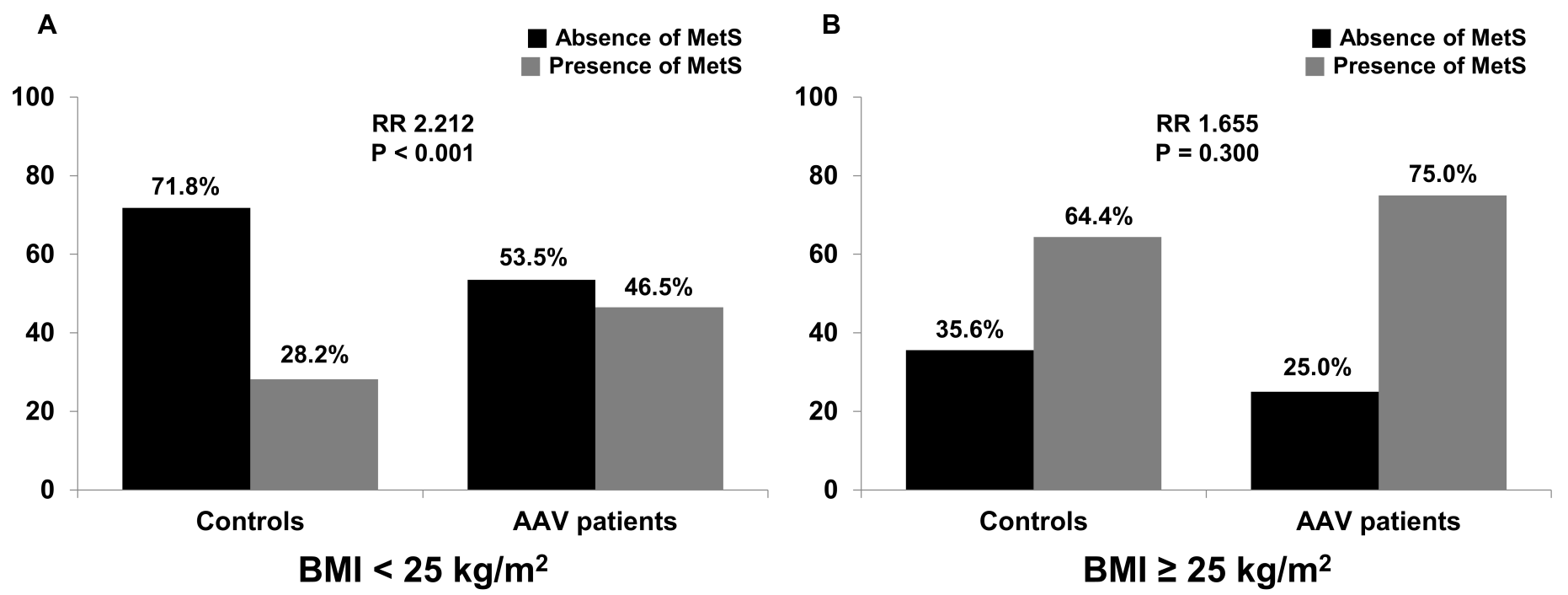

Figure 1

Relative risk of MetS in obese and nonobese subjects. Among nonobese subjects, the prevalence of MetS in nonobese AAV patients was significantly higher than that in nonobese controls (RR 2.212). Whereas, no difference in the prevalence of MetS was found between obese AAV patients and controls. MetS: metabolic syndrome, AAV: antineutrophil cytoplasmic antibody-associated vasculitis; RR: relative risk.

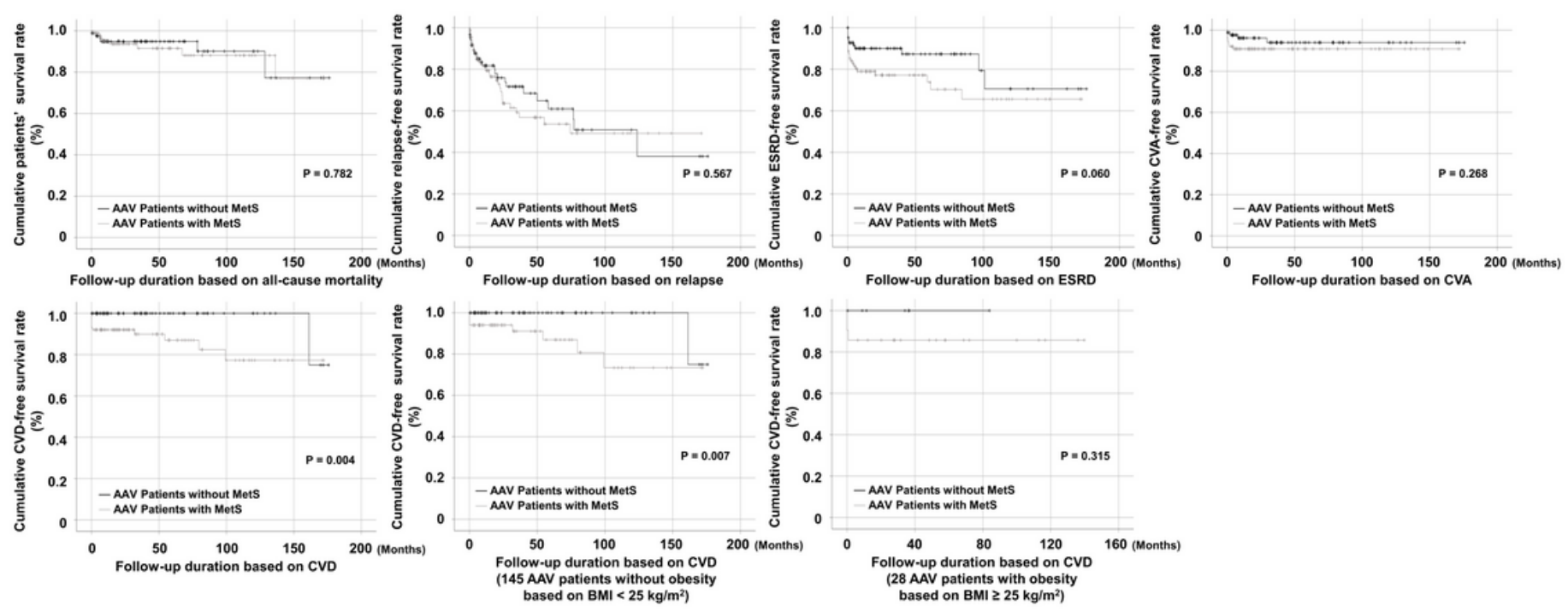

Figure 2

Cumulative survival rates based on MetS. Among the five poor outcomes of AAV, AAV patients with MetS exhibited the lower cumulative CVD-free survival rate than those without MetS during the follow-up period based 
on CVD. In addition, MetS at diagnosis significantly increased CVD occurrence only in nonobese AAV patients, but not in obese AAV patients. MetS: metabolic syndrome, AAV: antineutrophil cytoplasmic antibody-associated vasculitis; CVD: cardiovascular disease.

\section{Supplementary Files}

This is a list of supplementary files associated with this preprint. Click to download.

- SUPPLEMENTARYFIGURE1MetSAAV.tif

- SUPPLEMENTARYTABLE2MetSAAV.docx

- SUPPLEMENTARYTABLE1MetSAAV.docx 